\title{
Propuesta de producción de biodiésel mediante aceite vegetal usado
}

\section{Proposal to produce biodiesel using waste vegetable oil}

\author{
OSORIO-CANUL, Marvin†*, GARCÍA-MAGALLANES, Gabriel, HERRERA-LUGO, Angélica y \\ NOVELO-MOO, Thelma
}

\section{Universidad Tecnológica Metropolitana-Mérida}

ID $1^{\text {er }}$ Autor: Marvin, Osorio Canul / ORC ID: 0000-0002-6129-2559, Researcher ID Thomson: Marvin Efrain OsorioCanul, arXiv Author ID: Marvin-Osorio-Canul

ID $1^{\text {er }}$ Coautor: Gabriel, García Magallanes / ORC ID: 0000-0002-2091-6433, Researcher ID Thomson: Gabriel Iván García-Magallanes, arXiv Author ID: Gabriel-Magallanes

ID $2^{\text {do }}$ Coautor: Angélica, Herrera Lugo / ORC ID: 0000-0001-8066-8956, CVU CONACYT ID: 272950

ID $3^{\text {er }}$ Coautor: Thelma, Novelo Moo / ORC ID: 0000-0002-6383-9933, CVU CONACYT ID: 458937

DOI: $10.35429 / J R E .2019 .11 .3 .23 .28$

Recibido 18 de Octubre, 2019; Aceptado 12 Diciembre, 2019

\section{Resumen}

Actualmente, el uso de las energías renovables va en constante aumento, para ello, las universidades deben brindar a sus estudiantes una constante actualización con las técnicas innovadoras que permitan una compresión de los conceptos de la producción de biocombustibles, en este caso iniciaremos con la producción de biodiésel. En el presente trabajo se muestra la producción de biodiésel mediante la técnica de transesterificación. Esto con ayuda de equipos y materiales con los que cuenta la universidad, como un reactor químico. Este y otros equipos se estarán reactivando y reparando para el buen funcionamiento futuro. Con el fin de establecer las condiciones de producción acordes con el reactor químico, las instalaciones y equipos de la universidad, se han realizado pruebas con aceite vegetal nuevo para posteriormente escalar las condiciones al empleo de aceite vegetal usado. La importancia de producir biodiésel radica en la aportación de la reducción de las emisiones generadas por la combustión y darle un aprovechamiento al desperdicio del aceite vegetal.

Aceite vegetal usado, Biodiésel, Transesterificación

\begin{abstract}
Today, the use of renewable energies is constantly increasing, for that reason, Universities must constantly provide update to their students with innovative techniques that allow a compression of the concepts of biofuel production, in our case, will start with the production of biodiesel. The current document shows the production of biodiesel using the transesterification technique. All this with the help of equipment and materials available to the University, as a chemical reactor. This equipments will be reactivating and repairing for the good future functioning. In order to establish production condition chords to the chemical reactor, facilities and equipment of the university, has been tested with new vegetable oil to later scale the conditions to the use waste vegetable oil. The importance of producing biodiesel are in contribution of emission reduction from combustion and making use of the waste of vegetable oil.
\end{abstract}

Waste vegetable oil, Biodiesel, Transesterification

Citación: OSORIO-CANUL, Marvin, GARCÍA-MAGALLANES, Gabriel, HERRERA-LUGO, Angélica y NOVELOMOO, Thelma. Propuesta de producción de biodiésel mediante aceite vegetal usado. Revista de Energías Renovables. 2019. 3-11: $23-28$

\footnotetext{
* Correspondencia del Autor (Correo electrónico: marvin.canul.10@ hotmail.com)

$\uparrow$ Investigador contribuyendo como primer autor.
} 


\section{Introducción}

Viviendo en un tiempo en el que el calentamiento global se ha convertido en una prioridad principal, el uso de energías limpias en el mundo es un tema fundamental. El biodiésel es un biocombustible obtenido a partir de aceites vegetales, sus propiedades son similares al combustible diésel del petróleo, por ello, estos se pueden mezclar (SENER, 2018).

El aprovechamiento de desechos para producir combustibles puede resolver varios problemas a la vez, ya que permite reducir la contaminación que genera el vertido de estos residuos en los caños y desagües pluviales. Además, la generación de combustibles que pueden utilizarse en motores diésel, reduce las emisiones de $\mathrm{CO}_{2}$.

Desde el punto de vista económico, se genera una alternativa relacionada a la recolección del aceite vegetal usado y a su procesamiento posterior. Los datos obtenidos en varios países indican que el biodiésel obtenido de aceites reciclado de cocina probablemente no remplace al combustible diésel completamente; sin embargo, una sustancial cantidad de diésel puede ser mezclado con biodiésel preparado de aceites reciclado de cocina, ayudando en parte a disminuir la dependencia de combustibles basado en petróleo (Barriga Moreno, 2011).

La mayor parte $(75-85 \%)$ del costo de producción del biodiésel corresponde a la materia prima (grasa o aceite), por lo que las tecnologías encaminadas a uso y producción de aceites alternativos tendrían gran impacto, ya que los estudios ambientales indican que el uso de aceites y grasas residuales produce el biodiésel con las menores emisiones de gases de efecto invernadero. Sin embargo, sigue siendo un reto tecnológico el trabajar con materias primas de residuos, por lo que varias de nuestras acciones estratégicas van encaminadas a solucionarlo (Macera, 2011).

\section{Historia del biodiésel}

La técnica de transesterificación en aceites vegetales fue desarrollada en 1853 por los británicos E. Duffy y J. Patrick con el principal objetivo de obtener glicerina y utilizarla como materia prima en la producción de jabón, varios años antes de que se hubiera inventado el motor de biodiésel.
Esta técnica, primordialmente empleaba aceites de cacahuate, marihuana y maíz; actualmente se utilizan una gran cantidad de materias primas como son los aceites vegetales y también ha sido aplicada con éxito en grasas animales y aceite comestible residual (Balat \& Balat, 2008).

Hasta principios del siglo XX los motores automotrices utilizaban dos tipos de combustibles, los derivados del petróleo y los biocombustibles, pero en 1920 se impulsó a nivel internacional la penalización de la marihuana (principal materia prima de los biocombustibles por su bajo costo), lo que favoreció la explotación y comercialización de los derivados del petróleo y la tecnología que los ocupa, entre ellas el motor de diésel.

Lo que actualmente conocemos como biodiésel fue utilizado por primera vez en motores automotrices en Europa durante la segunda guerra mundial ante la escasez de derivados del petróleo durante ese periodo especial.

Pero no fue sino hasta finales de la década de los 70's y principios de los 80's que la preocupación por los altos precios del petróleo promovió una extensa experimentación de la técnica de obtención de biodiésel de una gran variedad de aceites vegetales y grasas animales en la búsqueda de fuentes alternativas de energía.

La comercialización mundial del biodiésel alcanzó 7,952 millones de galones (26.8 millones de toneladas) en 2015, siendo USA el líder en producción, seguido de Brasil, Indonesia, Alemania, Francia y Argentina, este último es el mayor exportador actualmente.

\section{Materia prima para el biodiésel}

La producción de biodiésel utilizando aceites vírgenes, tiene como principal inconveniente el alto costo de manufactura que se debe al elevado costo de los aceites de origen vegetal (soya, canola, etc.); a este se suma la crítica debido a la utilización de tierras de cultivo y materia prima, que en vez de ser destinadas para el consumo alimenticio se utilizan en la producción de biodiésel (Barriga Moreno, 2011). 
Una solución particular es la producción con aceites vegetal usados por ser menos costosos, y ya que estos suelen ser vertidos en los caños y desagüe pluviales, lo cual producen altos niveles de contaminación; situación que es frecuente en locales de comida que utilizan grandes cantidades de aceite en sus cocinas. Su uso permitirá aprovechar ese desecho y dar un uso a un producto contaminante, logrando así aminorar la contaminación ambiental (Ballesteros, 2008).

La importancia de producir biodiésel radica en la reducción de las emisiones generadas por la combustión. Con este procedimiento, se tienen reducciones netas de $\mathrm{CO} 2$ de $100 \%$, además de reducciones de SOx cercanas al $100 \%$ debido a la ausencia de Azufre en el biodiésel, de hollín entre 46-60\%; de CO entre 10-50\%; HC entre 10-50\%; y de aldehídos y compuestos poli-aromáticos en torno a $13 \%$.

\section{Producción de biodiésel en México}

En México la cadena de valor que opera es la producción de aceites usados y grasas. La producción comercial de biodiésel con aceites crudos no se ha podido sostener, debido a la falta de insumos a costos competitivos. Un de SENER (2016) en México, se han identificado 3 plantas de producción de biodiésel que llegaron a operar comercialmente y han cesado operaciones:

1. En Cadereyta, Nuevo León, utilizaba grasas animales (res y pollo) y aceites vegetales de desecho. Su capacidad instalada era de 18,000 m³/año. Dejó de operar en 2011.

2. En Lázaro Cárdenas, Michoacán, se instaló una planta anexa a una fábrica de aceite vegetal, con capacidad de 9,000 $\mathrm{m}^{3} / a n ̃ o$. Fue inaugurada en 2007 y debía utilizar Jatropha curcas e higuerilla; por falta de producción de esos cultivos, cerró en 2008.

3. En Chiapas en 2010, se instaló una planta de $10,000 \mathrm{~m}^{3} /$ año. Utilizaría Jatropha curcas, pero no hubo producción de semillas. Cerró operaciones en 2011.

La figura 1 muestra fotografías de las primeras dos plantas mencionadas.
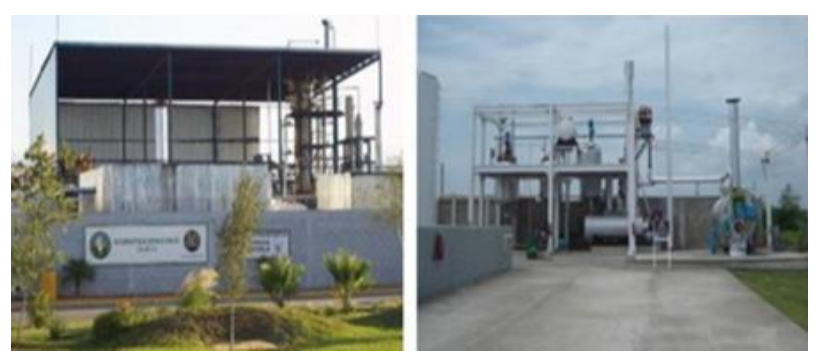

Figura 1 Plantas de producción de biodiésel en México. Caldereyta, Nuevo León (izquierda). Lázaro Cárdenas, Michoacán (derecha)

Fuente: SENER (2016)

México cuenta con 77 plantas generadoras, que representan el $1.3 \%$ de la capacidad total instalada (1,007 MW) del país y emplearon algún tipo de biocombustible para producir 1,884 GWh durante el 2017. Los estados de Veracruz, Jalisco y San Luis Potosí concentran el 53.9\% de la capacidad total de esta tecnología debido al aprovechamiento de los residuos orgánicos en los ingenios azucareros y del procesamiento de los residuos sólidos urbanos (SENER, 2018).

\section{Biodiésel}

La ASTM (American Society for Testing and Materials) define al biodiésel como ésteres monoalquílicos de cadena larga de ácidos grasos (FAME). Se encuentra en estado líquido y se obtiene a partir de recurso renovables como aceites vegetales de soja, colza/canola, girasol, palma y otros, como grasas animales y aceites usados, a través de un proceso denominado transesterificación (Garrido, 2010).

Se considera un combustible alternativo para motores de combustión interna. Generalmente el biodiésel está compuesto por ésteres metílicos de cadena larga $\left(\mathrm{C}_{14}-\mathrm{C}_{22}\right)$ de ácidos grasos como el ácido láurico, palmítico, esteárico y oleico, además de alcoholes de cadena corta, provenientes del metanol o el etanol.

El biodiésel es el mejor candidato para el gasóleo en los motores diésel. El biodiésel es producido principalmente a partir de aceite de soja, colza y aceite de palma (Demirbas, 2009).

La práctica internacional condujo a la adopción de una nomenclatura única para identificar la concentración de biodiésel en mezclas, conocida como nomenclatura BXX, donde XX corresponde al porcentaje en volumen del biodiésel en la mezcla diésel/biodiésel. 
Por ejemplo: B2, B5, B20, B100 son combustibles con una concentración de $2 \%, 5 \%$, $20 \%$ y $100 \%$ de biodiésel, respectivamente, Actualmente existen cuatro concentraciones principales de biodiésel que se utilizan en el mercado de los combustibles. El total (B100), las mezclas (B20 a B30), al aditivo (B5) y el aditivo lubricante (B2). Las mezclas B5 y B20 son las más comunes. La más utilizada actualmente es al $20 \%$ y cuando se utiliza como aditivo no supera el $5 \%$.

Las materias primas más utilizadas para la fabricación de biodiésel deben ser aquella que contenga un alto índice de triglicéridos como: aceite de girasol, colza, soja, aceite de frituras usados, sebo, etc. La tabla 1 muestra un resumen de las principales fuentes para la obtención de biodiésel.

\begin{tabular}{|l|}
\hline Aceites convencionales: \\
Girasol, Colza, Coco, Soja, Palma \\
\hline Aceites alternativos: \\
Brassica carinata (Colza etíope) \\
Cynara cardunculus (Cardo) \\
Camelina sativa (Sésamo bastardo) \\
Crambe abyssinica (Cambre) \\
\hline Otras fuentes: \\
Aceite de semillas modificadas genéticamente, Grasas \\
animales, Aceite de microalgas, Aceite de producciones \\
microbianas, Aceite de frituras. \\
\hline
\end{tabular}

Tabla 1 Principales materias primas para la elaboración de biodiésel

Fuente: Alfonso Álvarez (2013)

El objetivo de este estudio es establecer las condiciones de proceso de para obtener biodiésel a partir de aceite vegetal mediante una reacción de transesterificación y utilizando un reactor químico didáctico PIGNAT 02/2000.

\section{Metodología}

La puesta en marcha del reactor químico PIGNAT 02/2000, activando mangueras, partes; para el uso completo del equipo es la primera actividad para llevar a cabo la producción de biodiésel. Posteriormente se verifican las condiciones físicas del aceite vegetal usado adecuado para la obtención el biodiésel, que sirven para la obtención de las especificaciones de proceso adecuadas para la realización del biodiésel. Una vez obtenido el biodiésel se realiza una evaluación bajo diferentes condiciones físicas y parámetros establecidos que permitan realizar las pruebas del funcionamiento del biodiésel con un motor diésel.

\section{Adecuación del reactor}

Durante la verificación del reactor químico PIGNAT 02/2000 se observaron detalladamente las condiciones físicas de sus partes: mangueras, válvulas, tanque de doble pared para calentamiento, tanque de aceite de calentamiento, resistencias, sistema eléctrico, termómetros, medidores de caudal, dispositivos de fijación y estructura.

La estructura tubular, las mangueras que conectan el tanque de reacción con las etapas de separación compuestas de serpentines se encontraban en buenas condiciones físicas y adecuadas para su uso.

Sin embargo, se observó una ligera desalineación del tanque de reacción con la salida principal de los gases de reacción. Se procedió a solicitar la alineación al personal de mantenimiento de los laboratorios. También fue necesario el reemplazo del interruptor principal que estaba dañado y la limpieza de la resistencia del tanque de calentamiento de aceite.

\section{Caracterización del proceso}

La obtención del biodiésel se realizó mediante una reacción de transesterificación de $500 \mathrm{ml}$ aceite comestible de semillas de canola, calentado a $50{ }^{\circ} \mathrm{C}$, después se realizó una decantación para la separación los subproductos, seguido de un lavado y secado para la purificación del biodiésel.

\section{Resultados}

En la primera prueba se emplearon $140 \mathrm{ml} \mathrm{de}$ metóxido de sodio al $1.5 \% \mathrm{~m} / \mathrm{V}$ en alcohol etílico, calentándose durante $40 \mathrm{~min}$. Se pudo observar que además de la generación del biodiésel y la glicerina presencias de jabón.

En la figura 2 se pueden observar los productos de la reacción antes del proceso de separación y lavado. También se formó una pequeña cantidad de jabón y hubo presencia de la gran cantidad de agua atribuible a la baja calidad de alcohol y $\mathrm{NaOH}$ empleados. 


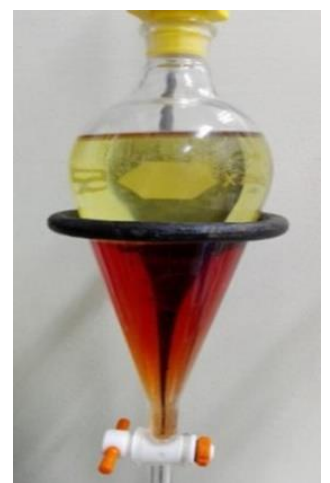

Figura 2 Productos obtenidos, biodiésel en la parte superior y glicerina de color marrón en la parte inferior Fuente: Elaboración Propia

Para las siguientes pruebas se empleó diferentes cantidades de metóxido de sodio y aumentando su concentración al $2.5 \% \mathrm{~m} / \mathrm{V}$ en alcohol etílico, con el objetivo de eliminar la formación de jabón. Para los lavados y secado de biodiésel se emplea la técnica de agitación mediante burbujeo y un secado por calentamiento durante 45-60 min. hasta la observación de un cambio de coloración propio del biodiésel. En las primeras se obtuvo biodiésel con una gran presencia de partículas de agua, ocasionando que el tiempo de secado previsto de 45-60 min aumentara a más de tres horas.

\section{Conclusiones}

Con la rehabilitación del reactor y la determinación de las condiciones de proceso, para la transesterificación de aceite vegetal tanto virgen como usado, proveniente de los comercios locales se pudo obtener un biodiésel que puede ser empleado como combustible.

\section{Referencias}

Alfonso Álvarez, J. A. (junio de 2013). Obtención de biodiésel a partir de aceites usados en casa habitación de la comunidad de Refugio. Chihuahua, Chihuahua, México.

Balat, M., \& Balat, H. (2008). A critical review of bio-diesel as a vehicular fuel. Energy Conversion and Management, 2727-2741. doi: 10.1016/j.enconman.2008.03. 016

Ballesteros Perdices, M. (julio de 2008). Los biocarburantes. España. Recuperado el 04 de enero de 2019, de http://webcache.googleusercontent.com/search? $\mathrm{q}=$ cache:http://imagenes.racc.es/pub/ficheros/ad juntos/adjuntos_mercedes_ballesteros_jzq_ed 9 4ed27.pdf
Barriga Moreno, E. A. (2011). Diseño del módulo de transesterificación de una planta piloto para producción de biodiésel a partir de aceites usados de cocina. Lima, Perú: Pontifica Universidad Católica de Perú.

Biodiésel y su situación en México y el mundo. (2017). Recuperado el 7 de enero de 2019, de Clúster biodiésel avanzado: http://bdavanzado.org/la-carrera-por-la-energiasolar-espacial/

Consuegra Pacheco, S. (2007). Guía de estudio y asignatura "Motores diésel". Colombia.

Demirbas, A. (2009). Progress and recent trends in biodiesel fuels. Energy conversion and Management, 14-34. doi: 10.1016/j.enconman.2008.09.001

Encinar, J. M., González, J. F., \& Rodríguez Reinares, A. (2005). Biodiesel from Used Frying Oil. Variables Affecting the Yields and Characteristics of the Biodiesel. Ind. Eng. Chem. Res., 5491-5499. doi:10.1021/ie040214f

Garrido, S. M. (2010). Producción de biodiésel a partir de aceites usados. Ciencias Sociales, 7586. Recuperado el 04 de enero de 2019, de file:///G:/Biodiesel/ DialnetTecnologiaTerritorioYSociedad -3319 142.pdf

J. M. (24 de marzo de 2018). Terrazocultor José Manuel. Recuperado el 13 de enero de 2019, de YouTube MX: https://www.youtube.com/watch?v=s71TliMb2 IQ

Kalligeros, S., Zannikos, F., Stournas, S., Lois, E., Anastopoulos, G., Teas, C., \& Sakellaropoulos, F. (2003). An investigation of using biodiesel/marine diesel blends on the performance of a stationary diesel engine. Biomass and Bioenergy, 141-149. doi:10.1016/S0961-9534(02)00092-2

Leclercq, E., Finiels, A., \& Moreau, C. (noviembre de 2011). Transesterification of Rapeseed Oil in the Presence of Basic Zeolites and Related Solid Catalysts. Journal of the American Oil Chemists' Society (JAOCS), 1161-1165.

Macera, Coralli, García, et al. (2011). La Bioenergía en México situación actual y perspectivas. Cuaderno Temático No. 4. Edición original publicada por Red Mexicana de Bioenergía, A.C.

OSORIO-CANUL, Marvin, GARCÍA-MAGALLANES, Gabriel, HERRERA-LUGO, Angélica y NOVELO-MOO, Thelma. Propuesta de producción de biodiésel mediante aceite vegetal usado. Revista de Energías Renovables. 2019. 
Meher, L. C., Dharmagadda, V. S., \& Naik, S. N. (2006). Optimization of alkali-catalyzed transesterification of Pongamia pinnata oil for production of biodiesel. Bioresource Technology, 1392-1397. doi: 10.1016/j.biortech. 2005.07.003

Meng, X., Chen, G., \& Wang, Y. (2008). Biodiesel production from waste cooking oil via alkali catalyst and its engine test. 851-857. doi: 10.1016/j.fuproc.2008.02.006

Narváez Rincón, P. C. (2010). Fuentes convencionales y no convencionales de energía: estado actual y perspectivas. Ingeniería e investigación, 165-173. Recuperado el 04 de enero de 2019, de https://www.redalyc.org/articulo.oa? $\mathrm{id}=643161$ 40016

Ramírez Coutiño, V. Á., Castañeda, M. E., Saldaña, L. G., \& González, L. M. (diciembre de 2017). Biodiésel a base de aceites usados. Revista de investigación y desarrollo, 3(10), 3944.

Saka, S., \& Kusdiana, D. (2001). Biodiesel fuel from rapeseed oil as prepared in supercritical methanol. Fuel, 225-331. doi:10.1016/S00162361(00)00083-1

SENER. (2006). Energías renovables para el desarrollo sustentable en México. México. Recuperado el 04 de enero de 2019, de http://awsassets.panda.org/

downloads/folletoerenmex_sener_gtz_isbn.pdf

SENER. (12 de 08 de 2016). Diagnóstico de la situación actual del biodiésel en México y escenarios para su aprovechamiento. México. Recuperado el 11 de 01 de 2019, de https://www.gob.mx/cms/

uploads/attachment/file/275444/Final_Report.p df

SENER. (2018). PRODESE. Programa de Desarrollo del Sistema Eléctrico Nacional. Recuperado el 01 de julio de 2019, de https://www.gob.mx/cms/uploads/attachment/fi le/331770/PRODESEN-2018-2032-

definitiva.pdf

Usta, N. (2005). Use of tobacco seed oil methyl ester in a turbocharged indirect injection diesel engine. Biomass and Bioenergy, 77-86. doi: 10.1016/j.biombioe.2004. 06.004
Wang, Y., Ou, S., Liu, P., Xue, F., \& Tang, S. (2006). Comparison of two different processes to synthesize biodiesel by waste cooking oil. Journal of Molecular Catalysis A: Chemical, 107-112. doi: 10.1016/j.molcata.2006.02.047 